\title{
Topological synthesis of non-fractionated differential geared mechanisms for automotives
}

\author{
D-Z Chen*, K-L Yao and J-J Lee \\ Department of Mechanical Engineering, National Taiwan University, Taipei, Taiwan
}

\begin{abstract}
In this paper, a methodology on the topological synthesis of admissible non-fractionated differential geared mechanisms for automotives is presented. Based on the characteristics of differential geared mechanisms, characteristics of non-fractionated differential geared mechanisms are laid out. Admissible non-fractionated differential geared mechanisms can be identified from atlases of geared kinematic chains by checking the redundancy condition in a kinematic equation governing the terminals. The methodology presented here is systematic and efficient, and can be easily applied to the enumeration of innovative non-fractionated differential geared mechanisms.
\end{abstract}

Keywords: automotives, non-fractionated differential geared mechanisms, topological synthesis

\section{INTRODUCTION}

The two-degree-of-freedom (DOF) differential geared mechanism (DGM) shown in Fig. 1a has been widely known and used in automotive drive systems. The automotive DGM is a geared mechanism with one actuator that distributes the input torque equally to its two outputs. Yan and Hsieh [1] synthesized the atlas of non-coupled two-DOF DGMs by assigning sun gears and planet gears on the admissible gear pair trees. Coupled two-DOF DGMs are constructed by joining the sun gears or carriers of two non-coupled DGMs into a set of coupling pairs. Hsu and $\mathrm{Wu}[2]$ identified the atlas of two-DOF DGMs with three terminals by adding a ground link to a set of coaxial links of one-DOF geared kinematic chains (GKCs). By defining the input and outputs in the DGM as terminals, Chen and Yao [3] developed a decomposition-based methodology for the topological synthesis of admissible $n$-terminal fractionated DGMs. They showed that an $n$-terminal fractionated DGM can be decomposed into an $(n-2)$-DOF GKC as the main component and a one-DOF mechanism as the input component. By identifying admissible input components and main components from the atlases of non-fractionated GKCs [4-7], admissible fractionated DGMs can be enumerated systematically by selecting a proper input component and a proper

The MS was received on 17 April 2002 and was accepted after revision for publication on 24 October 2002.

* Corresponding author: Department of Mechanical Engineering, National Taiwan University, No. 1 Roosevelt Road, Section 4, Taipei, Taiwan 10660.

C14602 C IMechE 2003 main component and sharing the connecting links of each component as a common link.

However, these researches are all focused on fractionated DGMs. The DGMs enumerated by Yan and $\mathrm{H}$ sieh [1], Hsu and $\mathrm{Wu}$ [2], and Chen and Yao [3] are only a special case of admissible DGMs. In this article, characteristics of $n$-terminal non-fractionated DGMs will be laid out based on the study of $n$-terminal DGMs. It will be shown that non-fractionated DGMs can be identified from atlases of geared kinematic chains by checking the redundancy condition in a kinematic equation governing the output terminals. Non-fractionated DGMs with three terminals are enumerated accordingly.

\section{CHARACTERISTICS OF $n$-TERMINAL DIFFERENTIAL GEARED MECHANISMS}

In graph representation, links are denoted by vertices, joints by edges, revolute joints by thin edges and gear pairs by heavy edges. Thin edges are labelled according to their axes in space. Figures $1 \mathrm{a}$ and $\mathrm{b}$ show the functional representation and a pseudo-isomorphic graph representation [6] of a standard automotive DGM. By defining the input together with the outputs as the terminal of a DGM, it can be seen that the automotive DGM shown in Fig. 1a is a three-terminal DGM. In Fig. 1a, link 1 is the input, links 2 and 3 are the outputs, while all the three terminals 1,2 and 3 are adjacent to link 0 , the ground. The three terminals are denoted by rectangles in Fig. 1b. 


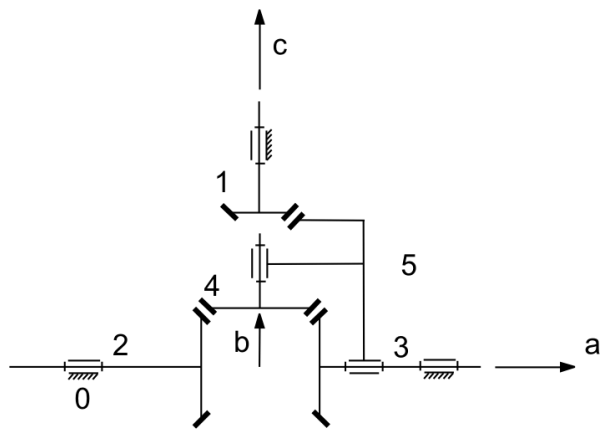

(a)

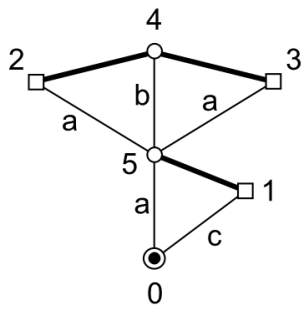

(b)

Fig. 1 The standard two-DOF DGM: (a) functional representation; (b) pseudo-isomorphic graph representation

From Fig. 1b, it can be seen that the number of links, $m$, the number of gear pairs, $J_{\mathrm{G}}$, and the number of revolute joints, $J_{\mathrm{R}}$, of the automotive DGM are six, three and five respectively. From Gruebler's equation, the DOF of the standard automotive DGM, F, can then be derived as

$$
\begin{aligned}
F & =3(m-1)-2 J_{\mathrm{R}}-J_{\mathrm{G}} \\
& =3 \times(6-1)-2 \times 5-3=2
\end{aligned}
$$

Fundamental circuits of the automotive DGM are $(2,4)(5),(3,4)(5),(1,5)(0)$, and their associated fundamental circuit equations $[\mathbf{8}]$ can be written as

$$
\left[\begin{array}{ccccc}
0 & 1 & 0 & -\gamma_{4,2} & \gamma_{4,2}-1 \\
0 & 0 & 1 & -\gamma_{4,3} & \gamma_{4,3}-1 \\
1 & 0 & 0 & 0 & -\gamma_{5,1}
\end{array}\right]\left[\begin{array}{l}
w_{1} \\
w_{2} \\
w_{3} \\
w_{4} \\
w_{5}
\end{array}\right]=0
$$

where $w_{i}$ denotes the angular velocity of link $i, \gamma_{i, j}=$ $\pm N_{i} / N_{j}$ denotes the gear ratio of the gear pair mounted on links $i$ and $j$ and the sign of the gear ratio is determined according to the positive rotation of link $i$ resulting in a positive or negative rotation of link $j$ along their predefined axes of rotation and $N_{i}$ is the number of teeth of gear $i$.

By suppressing $w_{4}$ and $w_{5}$ in equation (2), which are the angular velocities of non-terminals, the kinematic equation relating angular velocities of the terminals, $w_{1}$, $w_{2}$ and $w_{3}$, can be obtained as

$$
\left(\gamma_{3,2}-1\right) w_{1}+\gamma_{5,1} w_{2}-\gamma_{5,1} \gamma_{3,2} w_{3}=0
$$

where

$$
\gamma_{3,2}=\frac{\gamma_{4,2}}{\gamma_{4,3}}
$$

Hence, from equation (3), it can be seen that angular velocities of the three terminals are governed by one kinematic equation. From the extension of the characteristics of the standard automotive DGM, the general characteristics of $n$-terminal DGMs are derived and summarized as follows [3]:

$\mathrm{C} 1$. There is one kinematic constraint equation among terminals of an $n$-terminal DGM. The kinematic constraint equation can be written as

$$
\sum_{i=1}^{n} a_{i} w_{i}=0
$$

where $a_{i}$ is the coefficient and $w_{i}$ is the angular velocity associated with the $i$ th terminal.

C2. The $n$-terminal DGM is an $(n-1)$-DOF geared mechanism.

C3. All terminals in a DGM are adjacent to the ground link [9].

\section{CHARACTERIS TICS OF NON- FRACTIONATED DIFFERENTIAL GEARED MECHANISMS}

Although the admissible DGMs enumerated by Yan and $\mathrm{Hsieh}$ [1], $\mathrm{Hsu}$ and $\mathrm{Wu}$ [2], and Chen and Yao [3] are all fractionated DGMs, it is clear that any nonfractionated geared mechanism satisfying the characteristics of $n$-terminal DGM s as C1 to C3 are an admissible non-fractionated DGM. Note that an $n$-DOF nonfractionated geared mechanism is formed by holding one link of the GKC fixed. From C2, since an $n$-terminal DGM must be an $(n-1)$-DOF geared mechanism, then:

N1. The n-terminal non-fractionated DGM is an $(n-1)$-DOF non-fractionated geared mechanism, which can be formed by assigning one link of an $(n-1)$-D OF GKC as the ground link.

From C3, since all terminals of a DGM must be adjacent to ground, then:

N2. The ground link in an $n$-terminal non-fractionated DGM is adjacent to at least $n$ links. 
Let $g$ be the number of gear pairs of an $m$-link nonfractionated geared mechanism. Since the angular velocity of the ground is equal to zero, the fundamental circuit equations associated with the geared mechanism can be written in matrix form as

$$
\mathbf{A}_{g \times(m-1)} \cdot \mathbf{w}_{(m-1) \times 1}=0
$$

where $\mathbf{A}$ is a $g \times(m-1)$ matrix composed of gear ratios and $\mathbf{w}$ is an $(m-1) \times 1$ column matrix whose element is the angular velocity.

From $\mathrm{C} 1$, for a non-fractionated geared mechanism to be an admissible non-fractionated DGM, the kinematic constraint equation relating terminals has to be deduced from equation (6) by eliminating the angular velocities of the non-terminals. Hence, the columns associated with the non-terminals in matrix $\mathbf{A}$ must contain at least two non-zero elements such that the elements in matrix $\mathbf{A}$ associated with the non-terminal terms can be eliminated; otherwise, the non-terminal will be a redundant link in the DGM. Note that a link is considered to be a redundant link if either all of its associated gear pairs have no effect on the motions of every terminal or the corresponding gear ratios do not appear in the kinematic constraint equation. This leads to the following characteristic:

N3. The non-terminal(s) in a non-fractionated DGM is (are) associated with at least two fundamental circuits.

\section{IDENTIFICATION OF ADMISSIBLE NON- FRACTIONATED DIFFERENTIAL GEARED MECHANISMS}

Based on $\mathrm{N} 1$ to $\mathrm{N} 3$, admissible $n$-terminal nonfractionated DGMs can be identified from the atlases of non-fractionated GKCs $[\mathbf{6}, 7]$. The procedure of identification can be summarized as follows:

Step 1. Determine the ground link. In this step, a graph in the atlas of $(n-1)$-DOF GKCs is selected. For all vertices in the selected graph, one vertex adjacent to at least $n$ vertices via thin edges is presumed as the ground link.

For the purpose of demonstration, the creation of three-terminal seven-link non-fractionated DGMs is

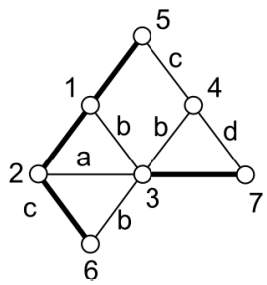

Fig. 2 GKC 5-7-2 used as an illustrative example. Admissible threeterminal seven-link non-fractionated DGMs can be identified from the atlas of two-DOF GKCs with seven links. From all 50 two-DOF seven-link GKCs [6], the GKC 5-7-2 is chosen as the illustrative example and is shown in Fig. 2. According to the coaxial conditions, link 1 in Fig. 2 is adjacent to three links as links 3, 4 and 6; link 3 is adjacent to four links as links 1, 2, 4 and 6; link 4 is adjacent to five links as links 1, 3, 5, 6 and 7; while link 6 is adjacent to three links as links 1, 3 and 4. Hence, links 1, 3, 4 and 6 in GKC 5-7-2 can be selected as the ground link.

Step 2. Determine the terminals and non-terminals. In this step, from all vertices adjacent to the ground link via thin edges, $n$ vertices are assigned as terminals. All vertices not being assigned as terminals are non-terminals. The graph is eligible to be a candidate of non-fractionated DGM if all nonterminals are associated with at least two fundamental circuits.

In GKC 5-7-2 shown in Fig. 2, link 4 is selected as the ground link for this case. Among the five links 1, $3,5,6$ and 7 adjacent to the ground link via thin edges, ten sets of terminals can be chosen from these five links as $(1,3,5),(1,3,6),(1,3,7),(1,5,6),(1,5$, $7),(1,6,7),(3,5,6),(3,5,7),(3,6,7)$ and $(5,6,7)$. For the purpose of demonstration, links 5, 6 and 7 are selected as the terminals in this case. Hence, the links not being selected as the ground link or the terminals, as links 1, 2 and 3, are assumed to be the non-terminals. Note that each of the three nonterminals 1,2 and 3 belongs to at least two fundamental circuits.

Step 3. Check redundancy. In this step, the kinematic constraint equation is derived by eliminating the items associated with non-terminals in the fundamental circuit equations. A link is viewed as a redundant link if all of the gear ratios of the corresponding gear pairs do not appear in the kinematic constraint equation. A candidate DGM with no redundant links is identified as an admissible $n$-terminal non-fractionated DGM.

The kinematic constraint equation among terminals 5, 6 and 7 of the GKC 5-7-2 is written as

$$
\gamma_{5,1} w_{5}-\gamma_{6,2} \gamma_{2,1} w_{6}+\gamma_{7,3}\left(\gamma_{6,2} \gamma_{2,1}-1\right) w_{7}=0
$$

Since the gear ratios of all four gear pairs exist in the kinematic constraint equation shown in equation (7), GKC 5-7-2 is identified as an admissible nonfractionated DGM with links 5, 6 and 7 as the terminals and link 4 as the ground link.

Step 4. Repeat steps 2 to 3 for each admissible ground link until all possible assignments of terminals and non-terminals are examined. The other nine sets of 


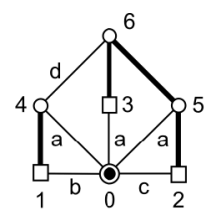

ND3-7-1

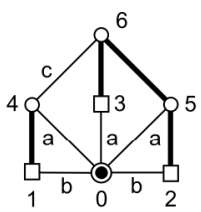

ND3-7-2

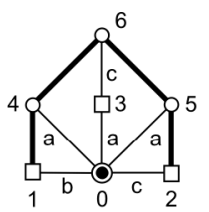

ND3-7-3

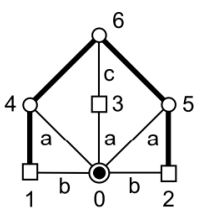

ND3-7-4

Fig. 3 Admissible three-terminal seven-link non-fractionated DGMs

terminals shown in step 2 are not available to be the terminals due to the existence of redundant links. For example, if links 1,3 and 6 are selected as the terminals, the kinematic constraint equation among terminals 1,3 and 6 is written as

$$
w_{1}+\left(\gamma_{6,2} \gamma_{2,1}-1\right) w_{3}-\gamma_{6,2} \gamma_{2,1} w_{6}=0
$$

Since the gear ratios $\gamma_{5,1}$ and $\gamma_{7,3}$ do not appear in equation (8), links 5 and 7 are redundant and GKC 5-7-2 is not an admissible non-fractionated DGM with links 1, 3 and 6 as the terminals.

Step 5. Repeat steps 1 to 4 until every admissible ground link is examined. Table 1 shows the identification results with different selection of the ground link and terminals of the GKC 5-7-2. In Table 1, it can be seen that the GKC 5-7-2 can only be an admissible non-fractionated DGM under the condition that links 5, 6, and 7 are chosen as the terminals and link 4 as the ground link.

Step 6. Exclude the isomorphic non-fractionated DGMs with the isomorphic detection techniques such as the Max and MinCodes [10] or the Degree Code [11]. Figure 3 shows the graph of admissible three-terminal non-fractionated DGMs with seven links identified from the atlas of two-DOF GKCs. In Fig. 3, terminals are represented with rectangles and

Table 1 The identification results with arrangements of the ground link and terminals of GKC 5-7-2

\begin{tabular}{llll}
\hline Ground link & Terminals & Redundant links & Identification result \\
\hline 1 & $3,4,6$ & 5,7 & - \\
3 & $1,2,4$ & $5,6,7$ & - \\
& $1,2,6$ & $4,5,7$ & - \\
& $1,4,6$ & 5,7 & - \\
& $2,4,6$ & $1,5,7$ & - \\
& & & - \\
4 & $1,3,5$ & $2,6,7$ & - \\
& $1,3,6$ & 5,7 & - \\
& $1,3,7$ & $2,5,6$ & - \\
& $1,5,6$ & $2,3,7$ & - \\
& $1,5,7$ & $2,3,6$ & - \\
& $1,6,7$ & 5 & - \\
& $3,5,6$ & 7 & ND3-7-1 \\
& $3,5,7$ & $1,2,6$ & - \\
\hline
\end{tabular}

$n$-terminal $m$-link non-fractionated DGMs are coded as NDn-m-\# with \# as the series number. Note that, in Fig. 3, ND3-7-1 shown in canonical form is linkrenumbered and is the non-fractionated DGM identified from GKC 5-7-2. Figures $4 a$ and $b$ show a possible functional representation of the nonfractionated DGMs ND3-7-2 and ND3-7-4 respectively. Since the DGMs enumerated by Yan and Hsieh [1], $\mathrm{Hsu}$ and $\mathrm{Wu}[2]$ and Chen and Yao [3] are all fractionated, all DGMs shown in Fig. 3 are believed to be new.

\subsection{Identification of three-terminal eight-link non- fractionated DGMs}

Figure 5 shows a GKC chosen from the atlas of twoDOF eight-link GKCs [7]. It can be seen that, by considering the coaxial conditions, links $0,1,2,3,4,5$ and 6 can be selected as the ground link. Seven cases can be derived by assigning alternative links as the ground link:

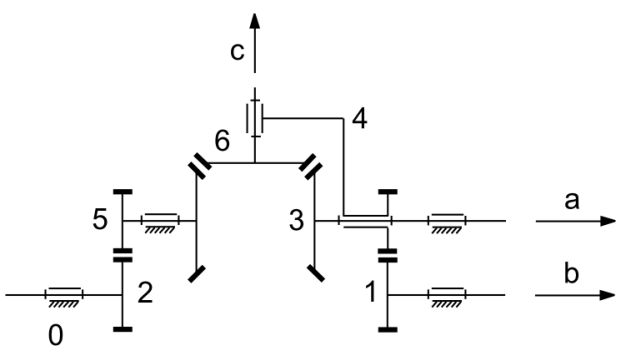

(a)

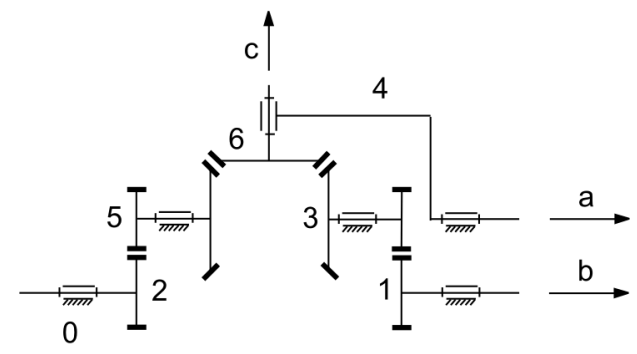

(b)

Fig. 4 (a) A possible design of ND3-7-2; (b) a possible design of ND3-7-4 


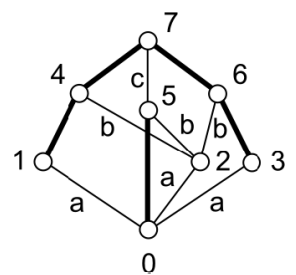

Fig. 5 A two-DOF eight-link GKC

1. Link 0 selected as the ground link. In this case, links 1,2 and 3 can be selected as the terminals. By eliminating the non-terminals in the fundamental circuit equations, the kinematic constraint equation governing terminals 1,2 and 3 can be derived as

$$
\begin{aligned}
& \gamma_{5,0} \gamma_{6,3} \gamma_{7,2} w_{1}+\left(\gamma_{4,1} \gamma_{5,0} \gamma_{7,4}-\gamma_{4,1} \gamma_{6,3} \gamma_{7,4}+\gamma_{4,1} \gamma_{6,3} \gamma_{7,6}\right. \\
& \left.-\gamma_{5,0} \gamma_{6,3} \gamma_{7,6}\right) w_{2}-\gamma_{4,1} \gamma_{5,0} \gamma_{7,4} w_{3}=0
\end{aligned}
$$

With no redundant link existing, it can be seen that the GKC can be an admissible non-fractionated DGM with link 0 selected as the ground link and links 1,2 and 3 selected as the terminals. The identified non-fractionated DGM is shown in Fig. 6 as ND3-8-17.

2. Link 1 selected as the ground link. In this case, links 0,2 and 3 can be selected as the terminals. By eliminating the non-terminals in the fundamental circuit equations, the kinematic constraint equation governing terminals 0,2 and 3 can be derived as

$$
\begin{aligned}
& \left(\gamma_{4,1} \gamma_{6,3} \gamma_{7,4}-\gamma_{4,1} \gamma_{6,3} \gamma_{7,6}\right) w_{0} \\
& \quad+\left(\gamma_{4,1} \gamma_{5,0} \gamma_{7,4}-\gamma_{4,1} \gamma_{6,3} \gamma_{7,4}+\gamma_{4,1} \gamma_{6,3} \gamma_{7,6}\right. \\
& \left.\quad-\gamma_{5,0} \gamma_{6,3} \gamma_{7,6}\right) w_{2}-\gamma_{4,1} \gamma_{5,0} \gamma_{7,4} w_{3}=0
\end{aligned}
$$

With no redundant link existing, it can be seen that the GKC can be an admissible non-fractionated DGM with link 1 selected as the ground link and links 0,2 and 3 selected as terminals. With renumbering and arranging the coaxial links into the canonical form, the identified non-fractionated DGM is shown in Fig. 6 as ND3-8-19.

3. Link 2 selected as the ground link. When link 2 is selected as the ground link, links $0,1,3,4,5$ and 6 can be selected as the terminals. Among the 20 sets of terminals, one non-fractionated DGM can be identified with links 0,1 and 3 selected as terminals. By eliminating the non-terminals in the fundamental circuit equations, the kinematic constraint equation relating terminals 0,1 and 3 can be obtained as

$$
\begin{aligned}
& \left(\gamma_{4,1} \gamma_{6,3} \gamma_{7,4}-\gamma_{4,1} \gamma_{6,3} \gamma_{7,6}\right) w_{0}+\gamma_{5,0} \gamma_{6,3} \gamma_{7,6} w_{1} \\
& \quad-\gamma_{4,1} \gamma_{5,0} \gamma_{7,4} w_{3}=0
\end{aligned}
$$

It can be seen that there is no redundant link in the non-fractionated DGM with link 2 selected as the ground link and links 0,1 and 3 as terminals. After renumbering and rearranging the coaxial links into the canonical form, the identified non-fractionated DGM is shown in Fig. 6 as ND3-8-18. The other 19 sets of terminals are not available to be the terminals due to the existence of redundant links.

4. Link 3 selected as the ground link. In this case, one non-fractionated DGM can be identified with links 0,1 and 2 selected as terminals. However, the DGM is isomorphic with ND3-8-19 and must be excluded.

5. Link 4 selected as the ground link. In this case, links 2, 5 and 6 can be selected as the terminals. By observing the gear ratio coefficient in the kinematic constraint equation, it can be seen that links 0,1 and 3 are redundant links. Hence, the GKC cannot be an admissible DGM with link 4 selected as the ground link.

6. Link 5 selected as the ground link. In this case, links 2, 4 and 6 can be selected as the terminals. Similarly, from the kinematic constraint equation, links 0,1 and 3 are identified as redundant links. Hence, the GKC cannot be an admissible DGM with link 5 selected as the ground link.

7. Link 6 selected as the ground link. In this case, links 2, 4 and 5 can be selected as the terminals. Following the same procedure for the redundancy check, it can be seen that links 0,1 and 3 are redundant links. Hence, the GKC cannot be an admissible DGM with link 6 selected as the ground link.

Figure 6 shows the graph representation of admissible three-terminal non-fractionated DGMs with eight links identified from the atlas of two-DOF GKCs [7]. With this methodology, admissible $n$-terminal non-fractionated DGMs can be efficiently identified from the atlases of $(n-1)$-DOF GKCs.

\section{CONCLUSION}

This article describes a systematic methodology for the topological synthesis of admissible $n$-terminal nonfractionated DGMs. In this approach, it is shown that non-fractionated DGMs can be identified from a procedure of checking whether a redundant link exists in the kinematic relation that governs the output terminals of a geared kinematic chain. Characteristics of admissible non-fractionated DGMs are also addressed. Atlases of admissible three-terminal nonfractionated DGMs are developed. The methodology presented here is completely general and can be easily applied to the conceptual design phase of nonfractionated DGMs. 


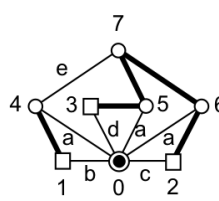

ND3-8-1

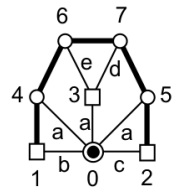

ND3-8-6

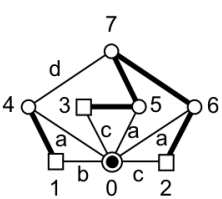

ND3-8-11

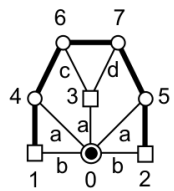

ND3-8-16

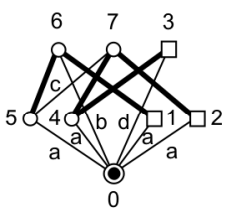

ND3-8-21

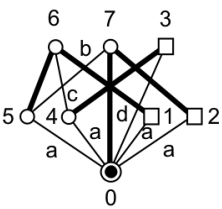

ND3-8-26

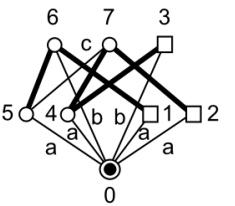

ND3-8-31

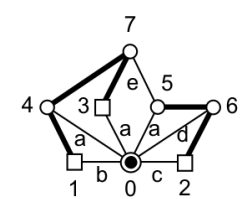

ND3-8-2

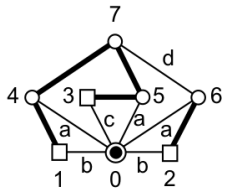

ND3-8-7

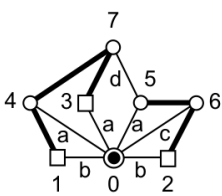

ND3-8-12

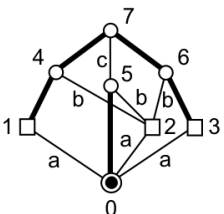

ND3-8-17

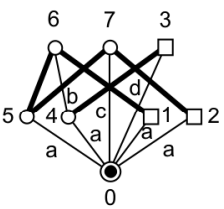

ND3-8-22

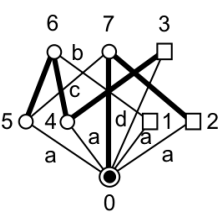

ND3-8-27

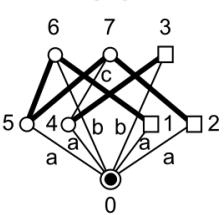

ND3-8-32
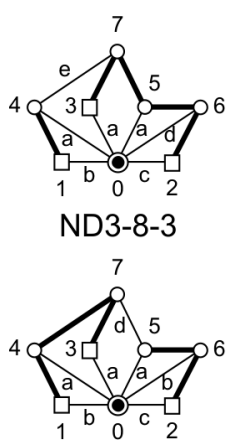

ND3-8-8

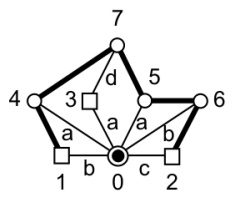

ND3-8-13

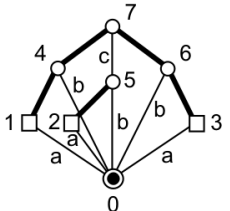

ND3-8-18

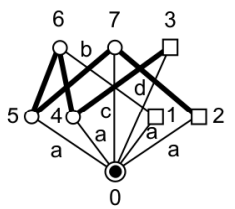

ND3-8-23

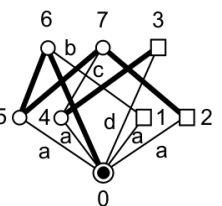

ND3-8-28

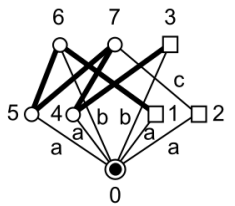

ND3-8-33
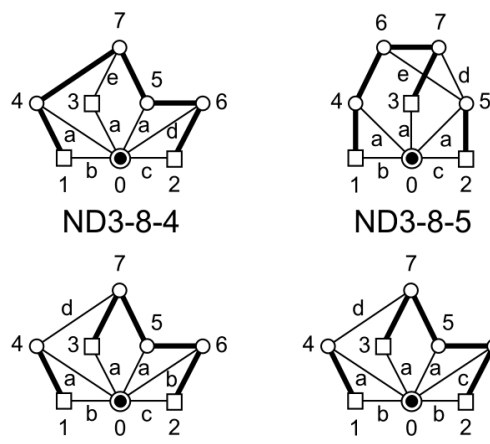

ND3-8-9

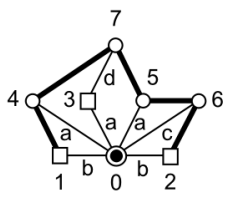

ND3-8-14

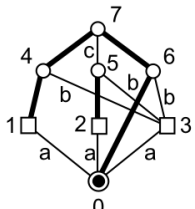

ND3-8-19

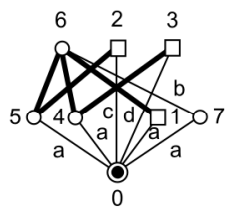

ND3-8-24

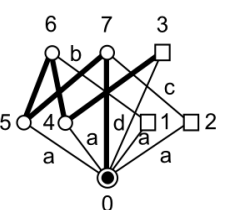

ND3-8-29

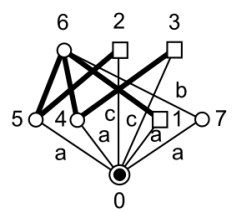

ND3-8-34

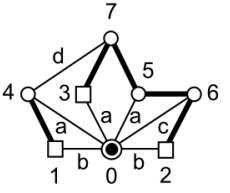

ND3-8-10

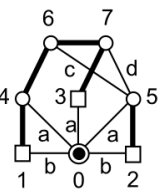

ND3-8-15

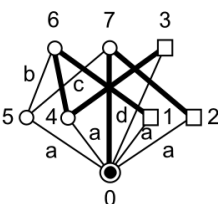

ND3-8-20

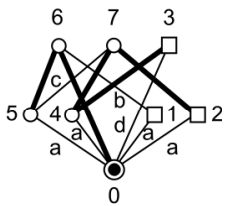

ND3-8-25

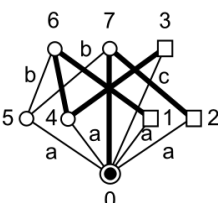

ND3-8-30

Fig. 6 Admissible three-terminal eight-link non-fractionated DGMs

\section{REFERENCES}

1 Yan, H. S. and Hsieh, L. C. Conceptual design of gear differentials for automotive vehicles. Trans. ASME, J. Mech. Des., 1994, 116, 565-570.

2 Hsu, C. H. and Wu, Y. C. A methodology for systematic synthesis of two-DOF gear differentials. Jap. Soc. Mech. Engrs Int. J., Ser. C, 1998, 41(2), 299-306.

3 Chen, D. Z. and Yao, K. L. Topological synthesis of fractionated geared differential mechanisms. Trans. ASME, J. Mech. Des., 2000, 122(4), 472-478.

4 Freudenstein, F. An application of Boolean algebra to the motion of epicyclic drives. Trans. ASME, J. Engng of Industry, Ser. B, 1971, 93, 176-182.

5 Tsai, L. W. An application of the linkage characteristics polynomial to the topological synthesis of epicyclic gear trains. Trans. ASME, J. Mechanisms, Transmission and Automn in Des., 1987, 109(3), 329-336. 
6 Tsai, L. W. and Lin, C. C. The creation of non-fractionated two degree-of-freedom epicyclic gear train. Trans. ASME, J. Mechanisms, Transmission and Automn in Des., 1989, 111, 524-529.

7 Hsu, C. H. On the structural synthesis and kinematic design of multi-DOF planetary gear trains (in Chinese). NSC Project Report NSC83-0401-E110-032, 1994.

8 Freudenstein, F. and Yang, A. T. Kinematics and statics of a coupled epicyclic spur-gear train. Mech. Mach. Theory, 1972, 7, 263-275.
9 Olson, D. G., Erdman, A. G. and Riley, D. R. Topological analysis of single-degree-of-freedom planetary gear trains. Trans. ASME, J. Mech. Des., 1991, 113, 10-16.

10 Ambekar, A. G. and Agrawal, V. P. Canonical numbering of kinematic chains and isomorphism problem: Min Code. Mech. Mach. Theory, 1987, 22(5), 453461.

11 Tang, C. S. and Liu, T. The Degree Code: a new mechanism identifier. Trans. ASME, J. Mech. Des., 1993, 115, 627-630. 